\title{
On The Optimality of Maisel Sidelobe Blanking Structure
}

\author{
Osman COŞKUN \\ Turkish Air Force \\ Ankara, Turkey \\ Email: osmanc@gmail.com
}

\author{
Çă̆atay Candan \\ Middle East Technical University (METU) \\ Department of Electrical and Electronics Engineering \\ Ankara, Turkey \\ Email: ccandan@metu.edu.tr
}

\begin{abstract}
We present an optimal sidelobe blanker (SLB) detector for Swerling-1 and Swerling-0 targets and compare the performances of the suggested detector with the classical Maisel SLB structure. The optimal SLB detector depends on the signal to noise ratio (SNR) and jammer to noise ratio (JNR) values and may not be practical for implementation in many applications. The goal of this work is to compare the Maisel structure with the optimal detector which utilizes additional information on target and jammer and assesses the performance gap between two systems. Numerical results show that the performance of Maisel SLB structure is close to the optimal detector under very practical conditions. ${ }^{1}$
\end{abstract}

\section{INTRODUCTION}

In the conventional radar systems, the interfering signals intercepted from the antenna sidelobes can cause problems such as false target detection and poor tracking accuracy etc To mitigate the effects of sidelobe signals, a sidelobe blanking (SLB) architecture is proposed by Maisel in [1]. In the Maisel structure, two receiving channels are used. The first one is the main channel whose antenna has high gain in main beam and low gain in the sidelobes. The second channel is called the auxiliary channel which has an omnidirectional pattern and has flat gain slightly greater than the sidelobe gain of the main antenna as shown in Fig. 1.

The blanking signal is generated when the ratio of the auxiliary channel output $(v)$ to main channel output $(u)$, that is $(v / u)$, is greater than blanking threshold $F$ as shown in Fig. 2 . When the ratio $v / u$ exceeds threshold $F$, the main channel is blanked. An erroneous blanking of the main channel results in a loss of detection probability.

It is easy to note from Fig. 1 that the gain of the omnidirectional antenna $\left(\omega^{2}\right)$ should satisfy the condition $\omega^{2} / \delta^{2}=\beta^{2} \geq$ 1 for a satisfactory operation. This condition can be easily justified by noting that an interfering signal in the sidelobe, say $u$; produces an auxiliary channel output of $\beta^{2} u$. To blank this signal, the condition of $\beta^{2} \geq F$ is trivially required, [1]. A similar consideration for the main channel, that is in order not to blank the main beam signal, the condition of $\omega^{2} \leq F$ is also necessary, [1].

The classical SLB systems are thoroughly studied in the literature. In [2], Farina examines the classical SLB system in

\footnotetext{
${ }^{1}$ This version of the paper contains some minor corrections over the version appearing Radar Conference, 2014 IEEE.
}

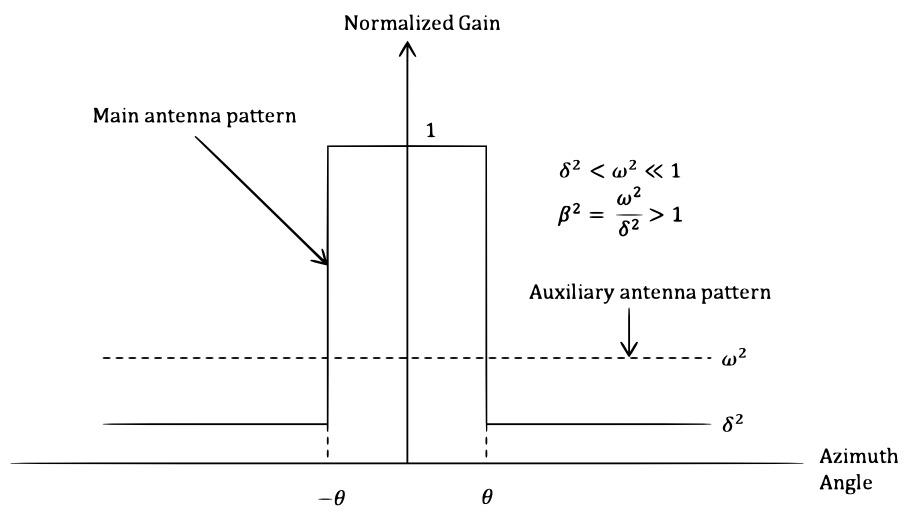

Fig. 1. Gain patterns of main and auxiliary antenna in SLB system

detail and derives the probability of blanking the jammer in sidelobe $\left(P_{b}\right)$, the probability of blanking the target in main beam $\left(P_{t b}\right)$, the probability of false target due to jammer in sidelobe $\left(P_{f t}\right)$ for Swerling-0 target model. In [3], Farina and Gini extends the aforementioned probability calculation to the Swerling-1 targets.

The classical SLB model is widely accepted and has been utilized in many systems. However, to the best of our knowledge, an optimality property of the classical SLB structure, in some sense, is not given in the literature. In [4], it is noted that the SLB systems derived from Neyman-Pearson likelihood ratio test (LRT) is hard to implement in real time and Maisel structure is suggested as a substitute with a simple implementation and an effective result.

In this work, we discuss an optimal SLB system for Swerling-1 and Swerling-0 targets and compare the performance of the optimal detector with the classical Maisel structure under different operating conditions. Numerical results suggest that the classical SLB system is close to the optimal system under highly practical operating conditions. Hence, the present work can be considered as a justification for the good performance of the Maisel structure.

\section{Proposed Sidelobe Blanking System For SWERLING-1 TARGETS}

Let $\tilde{s}$ and $\tilde{r}$ denote the complex valued matched filtered outputs of the main and auxiliary channels at a specific time. 


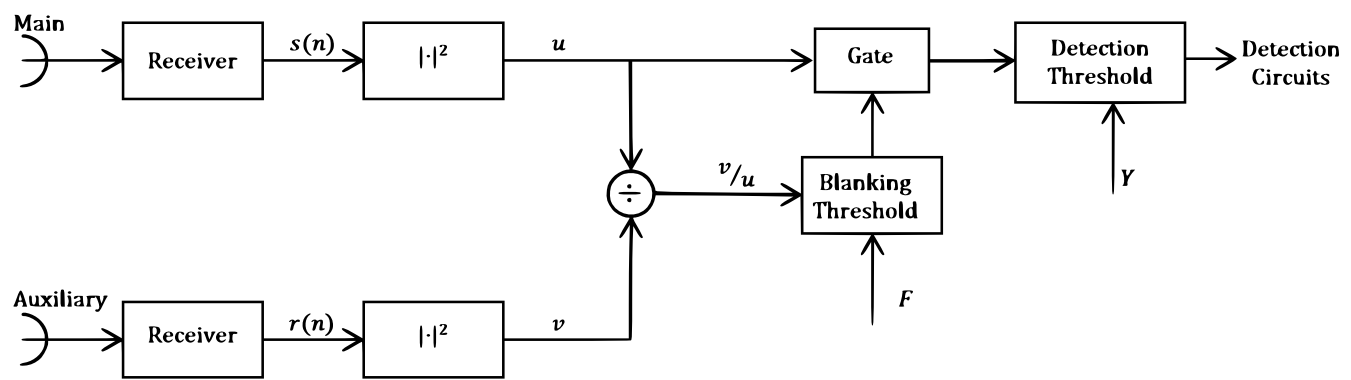

Fig. 2. Basic block diagram of classical SLB system

We have three hypotheses to choose: noise only $\left(H_{0}\right)$, target in main lobe and no jammer in sidelobe $\left(H_{1}\right)$, jammer in sidelobe and no target in main lobe $\left(H_{2}\right)$ :

$H_{0}:\left\{\begin{array}{l}\tilde{s}=\tilde{w}_{s} \\ \tilde{r}=\tilde{w}_{r}\end{array}, H_{1}:\left\{\begin{array}{l}\tilde{s}=\tilde{a}+\tilde{w}_{s} \\ \tilde{r}=\omega \tilde{a}+\tilde{w}_{r}\end{array}, H_{2}:\left\{\begin{array}{l}\tilde{s}=\tilde{c}+\tilde{w}_{s} \\ \tilde{r}=\beta \tilde{c}+\tilde{w}_{r}\end{array}\right.\right.\right.$

In (1), $\tilde{a} \sim \mathcal{C N}\left(0, \sigma_{a}^{2}\right)$ and $\tilde{c} \sim \mathcal{C N}\left(0, \sigma_{c}^{2}\right)$ indicate Swerling-1 target models [5] and $\tilde{w}_{s} \sim \mathcal{C N}\left(0, \sigma^{2}\right)$ and $\tilde{w}_{r} \sim \mathcal{C N}\left(0, \sigma^{2}\right)$ denote receiver noise in main and auxiliary channels, respectively. Here, $\mathcal{C N}\left(0, \sigma^{2}\right)$ represents zero mean complex circularly symmetric Gaussian random variables having the variance of $\sigma^{2}$. Note that by jammer, we mean an interfering target in sidelobe.

The parameters $\omega$ and $\beta$ appearing in (1) are the sidelobe gain of main antenna and gain of the auxiliary antenna (also see Fig. 1). Signal-to-noise (SNR) and jammer-to-noise ratios (JNR) are defined as:

$$
\begin{aligned}
& \mathrm{SNR}=\frac{E\left[|\tilde{a}|^{2}\right]}{E\left[\left|\tilde{w}_{s}\right|^{2}\right]}=\frac{\sigma_{a}^{2}}{\sigma^{2}}=\gamma_{s} \\
& \mathrm{JNR}=\frac{E\left[|\tilde{c}|^{2}\right]}{E\left[\left|\tilde{w}_{s}\right|^{2}\right]}=\frac{\sigma_{c}^{2}}{\sigma^{2}}=\gamma_{j}
\end{aligned}
$$

We note that the random variables $\tilde{r}$ and $\tilde{s}$ are correlated. The correlation under different hypotheses is as follows: $E\left[\tilde{r} \tilde{s}^{*} ; H_{1}\right]=\omega \sigma_{a}^{2}$ and $E\left[\tilde{r} \tilde{s}^{*} ; H_{2}\right]=\beta \sigma_{c}^{2}$. We introduce $\mathbf{x}=\left[\begin{array}{cc}\tilde{s} & \tilde{r}\end{array}\right]^{T}$ as a two dimensional Gaussian random vector with the correlation matrix $\mathbf{C}_{i}$ as follows:

$$
\mathbf{C}_{i}=E\left[\mathbf{x x}^{H} ; H_{i}\right]=\left[\begin{array}{ll}
E\left[|\tilde{s}|^{2} ; H_{i}\right] & E\left[\tilde{s} \tilde{r}^{*} ; H_{i}\right] \\
E\left[\tilde{s}^{*} \tilde{r} ; H_{i}\right] & E\left[|\tilde{r}|^{2} ; H_{i}\right]
\end{array}\right], i=\{1,2\}
$$

The probability density function (pdf) of $\mathrm{x}$ under $H_{i}$ becomes

$$
f_{\mathbf{X}}\left(\mathbf{x} ; H_{i}\right)=\frac{1}{\pi^{2}\left|\mathbf{C}_{i}\right|^{2}} \exp \left(-\mathbf{x}^{H} \mathbf{C}_{i}^{-1} \mathbf{x}\right), i=\{1,2\}
$$

and the covariance matrices $\mathbf{C}_{1}$ and $\mathbf{C}_{2}$ can be given as follows:

$$
\begin{aligned}
& \mathbf{C}_{1}=\sigma^{2}\left[\begin{array}{cc}
\gamma_{s}+1 & \omega \gamma_{s} \\
\omega \gamma_{s} & \omega^{2} \gamma_{s}+1
\end{array}\right], \\
& \mathbf{C}_{2}=\sigma^{2}\left[\begin{array}{cc}
\gamma_{j}+1 & \beta \gamma_{j} \\
\beta \gamma_{j} & \beta^{2} \gamma_{j}+1
\end{array}\right]
\end{aligned}
$$

The likelihood ratio test to discriminate $H_{1}$ and $H_{2}$ hypotheses can be written as:

$$
\Lambda(\tilde{r}, \tilde{s})=\frac{f_{\mathbf{X}}\left(\mathbf{x} ; H_{2}\right)}{f_{\mathbf{X}}\left(\mathbf{x} ; H_{1}\right)} \underset{H_{1}}{\stackrel{H_{2}}{\gtrless}} \zeta .
$$

Taking the logarithm of $\Lambda(\tilde{r}, \tilde{s})$ and ignoring non-data dependent terms, we reach the following test [6]:

$$
d=\mathbf{x}^{H}\left(\mathbf{C}_{1}^{-1}-\mathbf{C}_{2}^{-1}\right) \mathbf{x} \underset{H_{1}}{\stackrel{H_{2}}{\gtrless}} \eta
$$

The test consists of quadratic forms of complex Gaussian random variables.

The statistic of $\mathbf{x}^{H} \mathbf{Q} \mathbf{x}$ is important in several telecommunication applications [7]-[10]. Following the notation of [7] and [9], we define the $\mathbf{Q}$ matrix

$$
\mathbf{Q} \triangleq \sigma^{2}\left(\mathbf{C}_{1}^{-1}-\mathbf{C}_{2}^{-1}\right)=\left[\begin{array}{ll}
A & C \\
C & B
\end{array}\right]
$$

whose entries can be calculated through elementary algebra as

$$
\begin{aligned}
& A=\frac{\gamma_{s} \omega^{2}+1}{\gamma_{s} \omega^{2}+\gamma_{s}+1}-\frac{\gamma_{j} \beta^{2}+1}{\gamma_{j} \beta^{2}+\gamma_{j}+1}, \\
& B=\frac{\gamma_{s}+1}{\gamma_{s} \omega^{2}+\gamma_{s}+1}-\frac{\gamma_{j}+1}{\gamma_{j} \beta^{2}+\gamma_{j}+1}, \\
& C=-\frac{\gamma_{s} \omega}{\gamma_{s} \omega^{2}+\gamma_{s}+1}+\frac{\beta \gamma_{j}}{\gamma_{j} \beta^{2}+\gamma_{j}+1}
\end{aligned}
$$

The decision statistics $d$ in (4) can be expressed as follows:

$$
d=\mathbf{x}^{H} \mathbf{Q} \mathbf{x}=A|\tilde{s}|^{2}+B|\tilde{r}|^{2}+2 C \operatorname{Re}\left(\tilde{r} \tilde{s}^{*}\right)
$$

and its pdf can be written as follows, [7] and [8]:

$$
f_{d}(d)= \begin{cases}\frac{a b}{a+b} \exp (-a d) & d \geq 0 \\ \frac{a b}{a+b} \exp (b d) & d<0\end{cases}
$$

The parameters $a$ and $b$ appearing in (8) are defined through a rather complicated functions of $\mu_{\tilde{r} \tilde{s}}$ and $r$, [9]:

$$
\begin{aligned}
& a=\sqrt{r^{2}+\frac{1}{4\left(\mu_{\tilde{r} \tilde{r}} \mu_{\tilde{s} \tilde{s}}-\left|\mu_{\tilde{s} \tilde{r}}\right|^{2}\right)\left(|C|^{2}-A B\right)}}-r \\
& b=\sqrt{r^{2}+\frac{1}{4\left(\mu_{\tilde{r} \tilde{r}} \mu_{\tilde{s} \tilde{s}}-\left|\mu_{\tilde{s} \tilde{r}}\right|^{2}\right)\left(|C|^{2}-A B\right)}}+r
\end{aligned}
$$


where $\mu_{\tilde{r} \tilde{s}}=\frac{1}{2} E\left[\tilde{r} \tilde{s}^{*}\right]$ and

$$
r=\frac{A \mu_{\tilde{r} \tilde{r}}+B \mu_{\tilde{s} \tilde{s}}+C^{*} \mu_{\tilde{s} \tilde{r}}^{*}+C \mu_{\tilde{r} \tilde{s}}}{4\left(\mu_{\tilde{r} \tilde{r}} \mu_{\tilde{s} \tilde{s}}-\left|\mu_{\tilde{s} \tilde{r}}\right|^{2}\right)\left(|C|^{2}-A B\right)} .
$$

Threshold Calculation: The threshold $\eta$ for the NeymanPearson test can be easily calculated from (8). For a given target blanking probability $P_{t b}$ (declaring $H_{2}$ when $H_{1}$ is true), the threshold $\eta$ appearing in $P_{t b}=\operatorname{Pr}\left(H_{2} \mid H_{1}\right)=$ $\int_{\eta}^{\infty} f_{d \mid H_{1}}(x) d x$ can be written as follows:

$$
\eta= \begin{cases}-\frac{1}{a} \ln \left[\left(\frac{a+b}{b}\right) P_{t b}\right] & P_{t b} \geq \frac{b}{a+b} \\ \frac{1}{b} \ln \left[-\left(\frac{a+b}{a}\right)\left(P_{t b}-1\right)\right] & P_{t b} \leq \frac{b}{a+b}\end{cases}
$$

Blanking Probability Calculation: Using the threshold $\eta$, one can find the probability of blanking the jammer in sidelobe, $P_{b}=\operatorname{Pr}\left(H_{2} \mid H_{2}\right)=\int_{\eta}^{\infty} f_{d \mid H_{2}}(x) d x$, as follows:

$$
P_{b}= \begin{cases}\frac{b}{a+b} \exp (-a \eta) & \eta \geq 0 \\ \frac{a}{a+b}(1-\exp (b \eta))+\frac{b}{a+b} & \eta \leq 0\end{cases}
$$

This completes the derivation of main results for the mentioned SLB system.

Comments: We present a quantitative critique of the suggested detector and discuss the practical issues about its implementation. The optimal test for Swerling-1 targets given in (4) depends on several parameters including operating SNR and JNR. Hence, the optimality, in the sense of NeymanPearson, is achieved through the knowledge target and jammer specific parameters which are not utilized in the classical Maisel SLB system. Hence, the performance superiority of the suggested detector (if any) can be attributed to this additional knowledge. In several applications, it is not possible to reliably estimate SNR and JNR values on-the-fly and resorting to classical Maisel structure is unavoidable. Yet, the performance gap between the the optimal and Maisel structures, in spite of the unavailable information for the conventional structure, can be of interest and examined in this study.

In the following section, we present a numerical comparison of Maisel structure and the optimal detector. It is assumed that both systems are equipped with an antenna having identical $\omega$ and $\beta$ values. Both detectors are adjusted to meet a given target blanking (false blanking) probability. We would like to reiterate that the optimal detector uses both SNR and JNR in the detector design and the threshold calculations depend on both parameters for the optimal detector. Fig. 3 shows the dependency of the threshold $\eta$ on the JNR value for a practical operational scenario. It can be noted that the dependence of $\eta$ on JNR is quite weak for sufficiently large JNR values, say for JNR $>15 \mathrm{~dB}$. It should be noted that the Maisel structure does not have any dependence on JNR for the calculation of the threshold $F$.

Fig. 4 presents the receiver operating characteristics (ROC) curve for the suggested detector. ROC curve is given for target having SNR of $5 \mathrm{~dB}$ and the associated $P_{t b}$ and $P_{b}$ values for different JNR values are given. It can be noted that from this figure, dependency of $P_{b}$ on JNR is quite weak for JNR $>15$ dB.

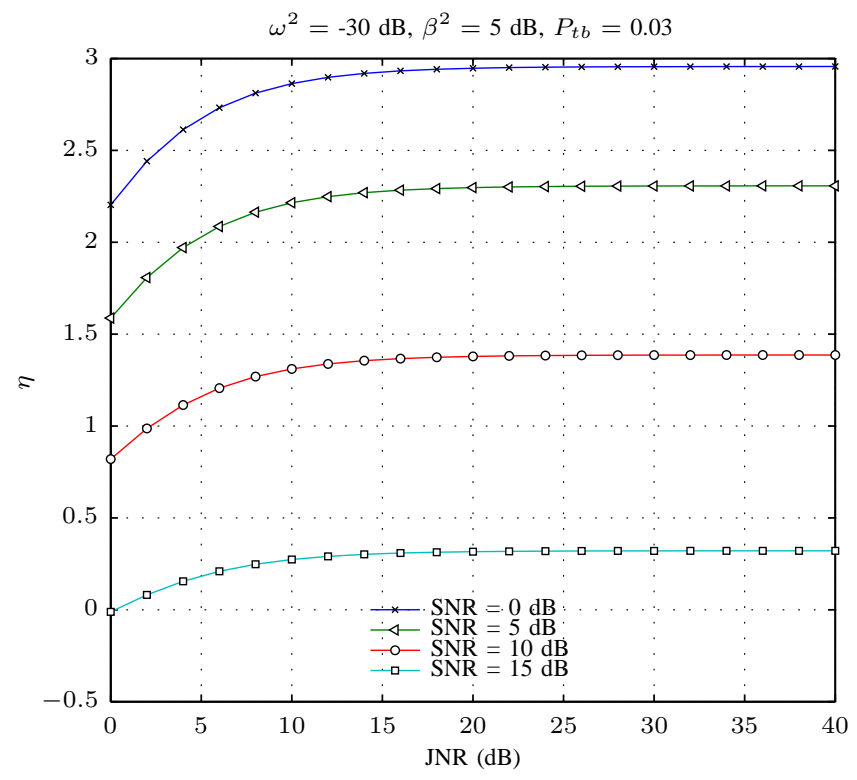

Fig. 3. Dependency of $\eta$ on JNR for the suggested detector (Swerling-1 target).

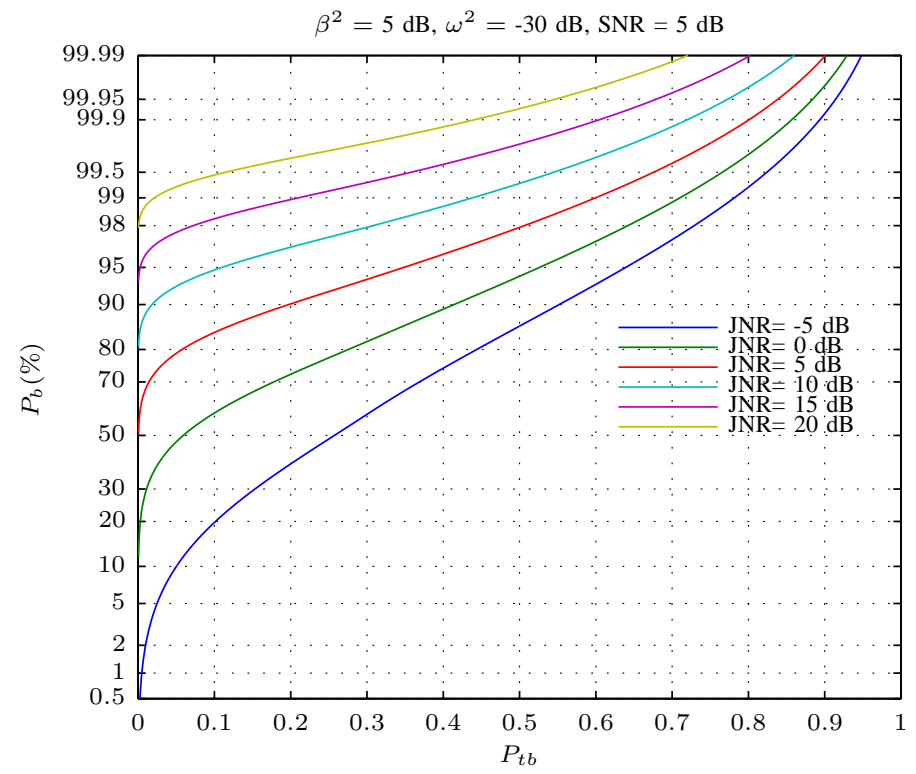

Fig. 4. Jammer blanking probability $P_{b}$ vs target blanking probability $P_{t b}$ of the suggested detector (Swerling-1 target).

\section{Numerical COMPARISONS With The MAisel STRUCTURE FOR SWERLING-1 TARGETS}

Setting threshold for a given $\mathbf{P}_{\text {tb }}$ and SNR: The undesired event for a SLB system is the blanking of a target 


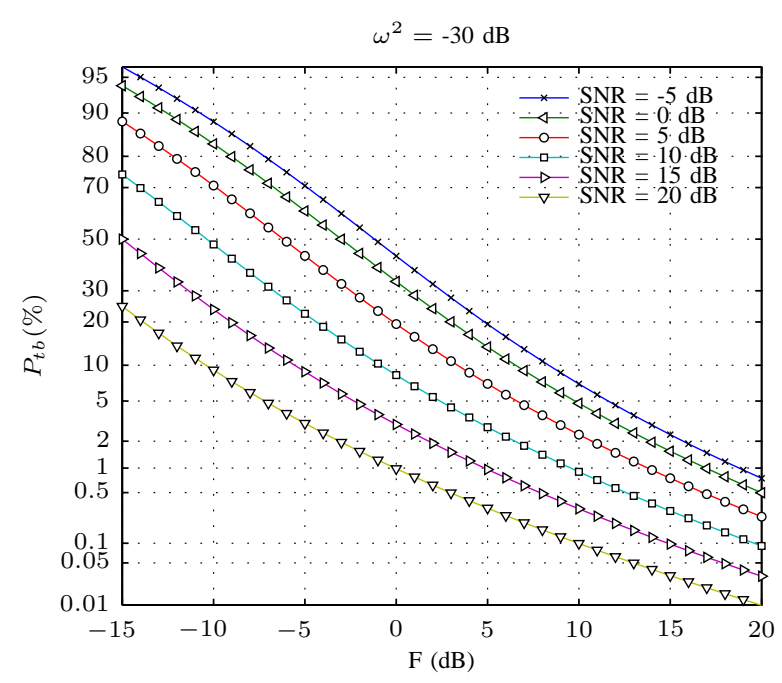

(a) Maisel SLB system (from [3])

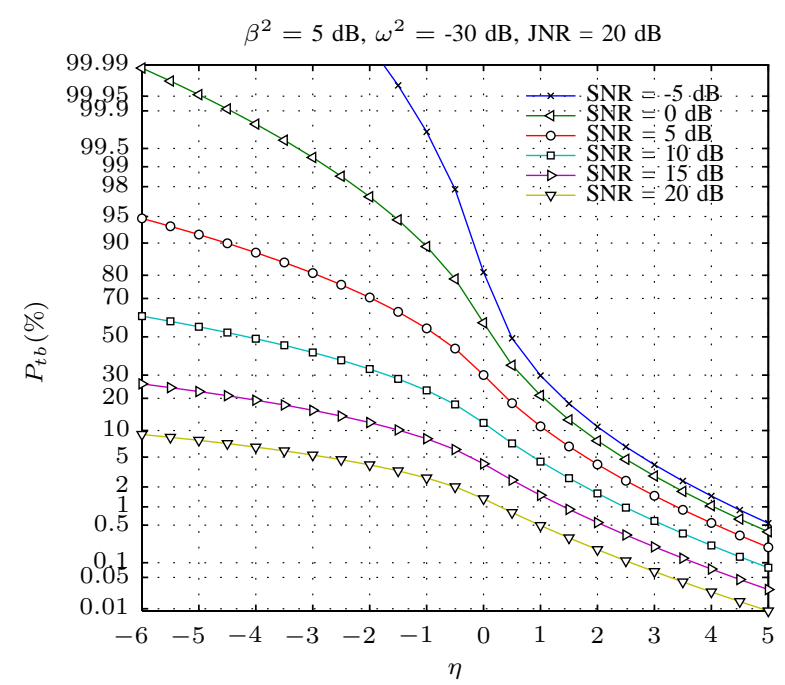

(b) Suggested detector

Fig. 5. Target blanking probabilities vs F and $\eta$ for Maisel and suggested SLB detectors (Swerling-1 target).

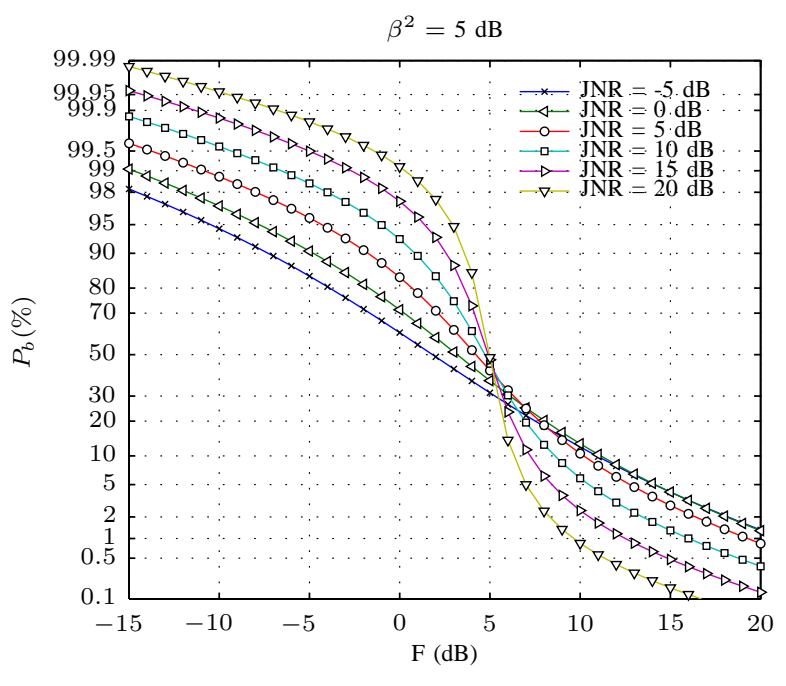

(a) Maisel SLB system (from [3])

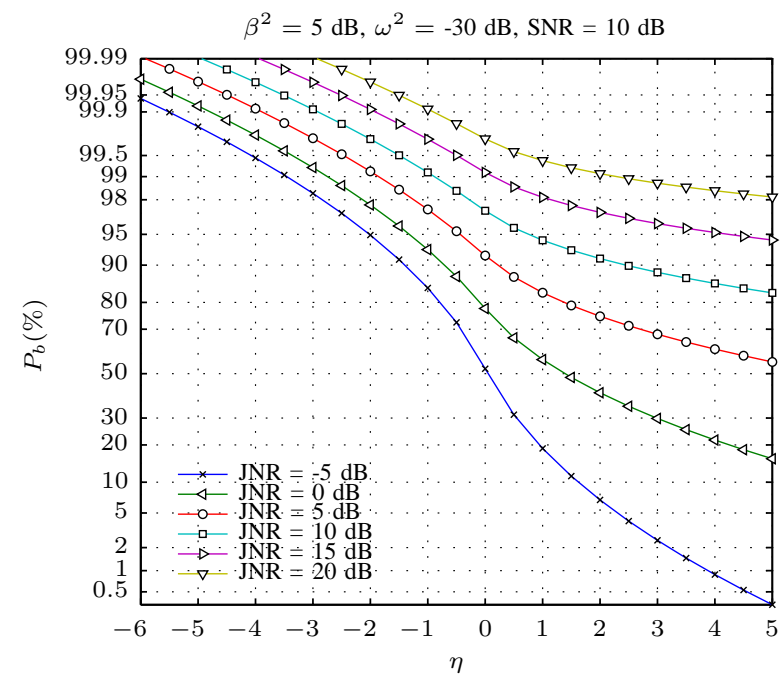

(b) Suggested detector

Fig. 6. $P_{b}$ vs $F$ and $P_{b}$ vs $\eta$ for the classical and suggested SLB detectors (Swerling-1 target).

presiding in the main-lobe when the jammer not present. Fig. 5 can be utilized to set the threshold values for both detectors at a given $P_{t b}$ and fixed SNR. As a practical remark, we would like to note that SNR value in the threshold calculation should be selected for the weakest detectable targets, that is for the targets barely crossing the detection threshold. It can be noted from this figure that targets having the potential of crossing the detection threshold with a significant margin (high SNR targets) have a smaller probability of getting blanked. As a side note, in Fig. 5(b), JNR $=20 \mathrm{~dB}$ is used when constructing $\mathbf{Q}$ matrix of the optimal detector.

Variation of $\mathbf{P}_{\mathbf{b}}$ with respect to JNR: The desired event for a SLB system is the blanking of jamming residing in the side-lobe. Fig. 6 shows the variation of this probability versus threshold for different JNR values. This figure can be utilized, along with Fig. 5, to examine the blanking performance of the system at a fixed probability of undesired event of false blanking.

It can be noted from Fig. 6(a) that $F$ values greater than $\beta^{2}=5 \mathrm{~dB}$ results in a significant loss of blanking probability irrespective of JNR value. This loss is quite expected since the condition of $F<\beta^{2}$ is violated. In contrast, the change of $P_{b}$ with respect to $\eta$ and JNR is rather smooth for the optimal detector.

Variation of $P_{b}$ with respect to $J N R$ for a given $\mathbf{P}_{\text {tb }}$ : Figure 7 (a) compares the performance of two systems at a fixed probability of target blanking. The target blanking probability is set to 0.01 in Fig. 7(a). The threshold values for each detector at different SNR values are denoted in the figure legend. $\mathrm{FNR}=20 \mathrm{~dB}$ is utilized for the threshold caleutation 


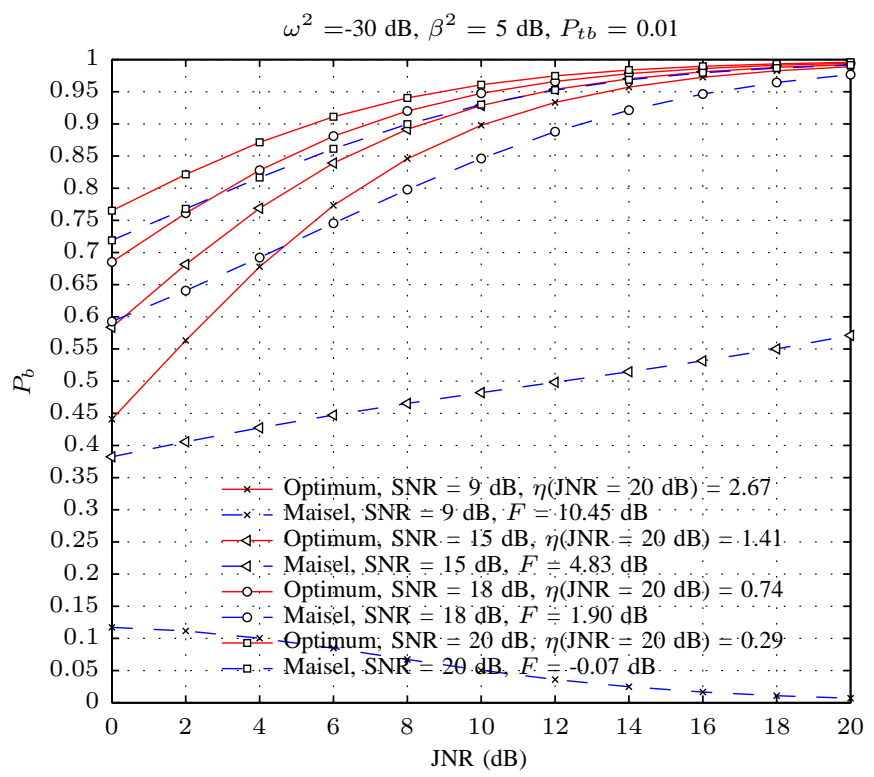

(a) $P_{t b}=0.01$

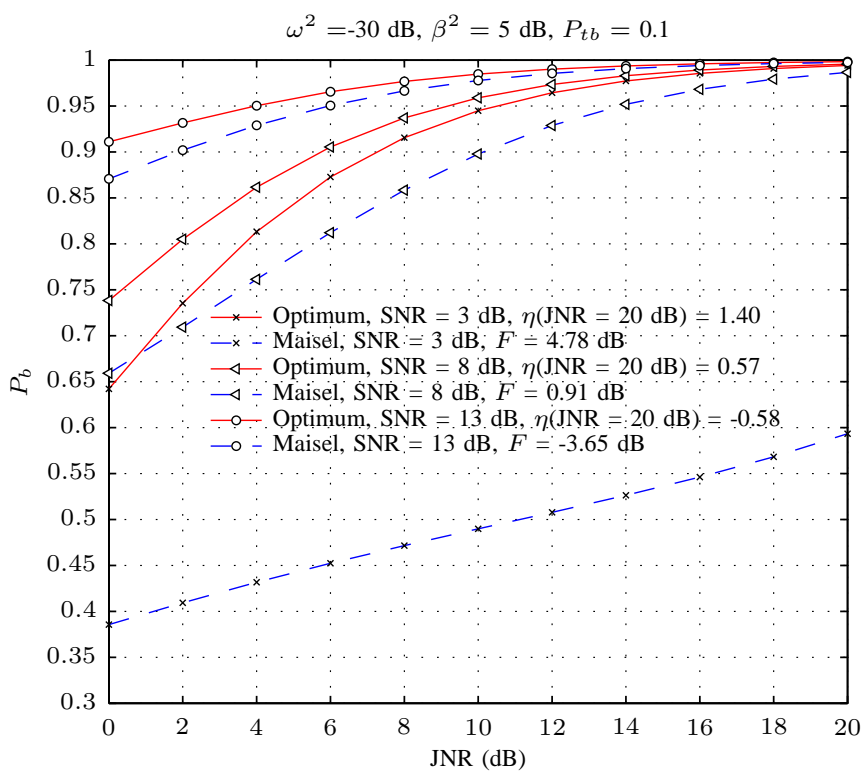

(b) $P_{t b}=0.1$

Fig. 7. Comparison of $P_{b}$ on JNR for Swerling-1 targets. Parameters: $\beta^{2}=5$ $\mathrm{dB}, \omega^{2}=-30 \mathrm{~dB}$

\section{of the optimaldeter.}

It can be noted from Fig. 7(a) that the Maisel structure behaves very poorly in two cases shown. Both of these cases correspond the case of having the threshold $F$ exceeding or being very close to $\beta^{2}$. As noted before, these cases are in violation of the condition $F<\beta^{2}$. The other cases have much higher blanking probability; but have a poorer performance in comparison to the optimal detector. It can be noted that the performance gap gets smaller as the threshold $F$ of the Maisel structure gets smaller in comparison to $\beta^{2}$.

Fig. 7(b) presents the result of an identical comparison for a higher target blanking of $P_{t b}=0.1$. For the given $P_{t b}$ value, the problematic cases of $F \approx \beta^{2}$ occurs at much smaller SNR values, i.e. $\mathrm{SNR} \approx 3 \mathrm{~dB}$. It can be noted that the cases for which the condition $F \ll \beta^{2}$ is satisfied, the performances of Maisel structure and the optimal detector are very similar.

\section{Optimum SLB System FOR SWERLing-0 TARgets AND ITS COMPARISON WITH MAISEL STRUCTURE}

Swerling-0 target model for the complex return signals $\tilde{a}$ and $\tilde{c}$ given in (1) assumes that the magnitude of $\tilde{a}$ and $\tilde{c}$ are constant and deterministic, but the phase is uniformly distributed on $(0,2 \pi)$.

As in Swerling-1 target model, $\tilde{r}$ and $\tilde{s}$ are correlated. The correlation under different hypotheses is as follows: $E\left[\tilde{r} \tilde{s}^{*} ; H_{1}\right]=\omega|\tilde{a}|^{2}$ and $E\left[\tilde{r} \tilde{s}^{*} ; H_{2}\right]=\beta|\tilde{c}|^{2}$. To obtain the joint pdfs of $\tilde{r}$ and $\tilde{s}$, we first write the conditional joint pdf given the phase is deterministic. After that, we integrate the result with respect to phase over $(0,2 \pi)$ and obtain joint pdfs. The ratio of joint pdfs are calculated as in (3) to obtain the optimum test. Due to space limitation, we include only resultant LRT which is

$$
d_{0}=\frac{I_{0}\left(\frac{2|\tilde{c}|}{\sigma^{2}}|\tilde{s}+\beta \tilde{r}|\right)}{I_{0}\left(\frac{2|\tilde{a}|}{\sigma^{2}}|\tilde{s}+\omega \tilde{r}|\right)} \stackrel{H_{2}}{H_{1}} \eta_{0}
$$

where $I_{0}(\cdot)$ is the modified Bessel function of the first kind.

The test given in (12) is optimum for Swerling-0 target. The statistic of the test, $d_{0}$ is analytically difficult to obtain. So we resort to Monte Carlo method for the performance assessment. The threshold $\eta_{0}$ is determined by generating $H_{1}$ hypothesis (signal plus noise) and searching the threshold which satisfies the predetermined false blanking probability $\left(P_{t b}\right)$.

Fig. 8 shows the threshold $\eta_{0}$ dependence on JNR for several values of SNR. The behaviour of $\eta_{0}$ on JNR is different when compared with Swerling-1 target. The threshold value strongly depends on JNR, but is almost independent of SNR for the values greater than $10 \mathrm{~dB}$.

Fig. 9(a) presents the $P_{b}$ comparison when the false blanking probability $P_{t b}$ is set to 0.01 . The corresponding threshold values are shown in figure legend. As in Swerling-1 case, Maisel structure behaves poorly when $F$ is not sufficiently smaller than $\beta^{2}$. It can be noted that, the performance gap gets smaller when JNR increases.

Fig. 9(b) shows the identical comparison when the false blanking probability is set to higher value of 0.05 . The comments about Fig. 7(b) are still applicable.

It can be commented that optimum SLB structure achieves the high blanking probability at a relatively small JNR values when compared with Swerling-1 case. This is expected due to assumption of non-fluctuating target model.

\section{CONCLUSiON}

The goal of this study is to justify the performance of the conventional Maisel side-lobe blanking structure. To this aim, an optimal detector is constructed for Swerling-1 and 


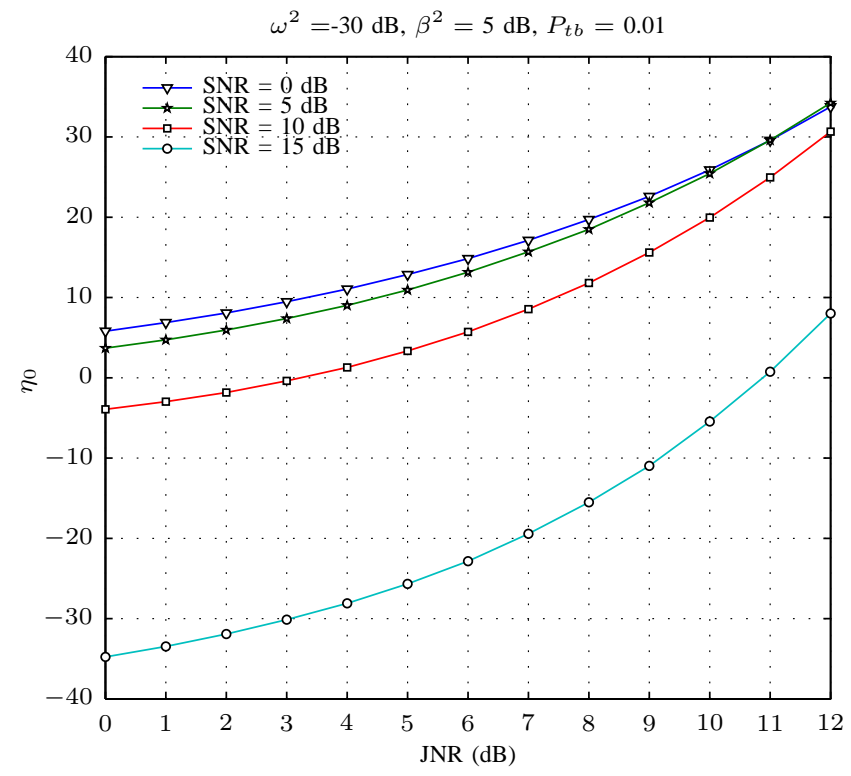

Fig. 8. Dependency of $\eta_{0}$ on JNR for the suggested detector (Swerling-0 target) (\# of Monte Carlo trials $=10^{6}$ ).

Swerling-0 target models. The statistics of optimum SLB detector for Swerling-1 targets is derived analytically. The optimal detector requires the knowledge of SNR and JNR values which is typically not known by the radar receiver. The main goal of this study is to examine the performance gap between the optimal receiver and Maisel structure in spite of the non-availability of the additional information for the optimal receiver.

The numerical results show that the Maisel structure performs in close proximity to the optimal detector when the operating threshold of Maisel structure $(F)$ is sufficiently smaller than $\beta^{2}$ which is the running assumption for a reliable operation with the Maisel structure in many applications.

A potential future work is the examination of detector sensitivity to SNR and JNR values in order to better understand the implementation feasibility of the optimal system. Another future work is the study of the optimal SLB system on the detection and false alarm probability of the overall system.

\section{REFERENCES}

[1] L. Maisel, "Performance of sidelobe blanking systems," Aerospace and Electronic Systems, IEEE Transactions on, vol. AES-4, no. 2, pp. 174$180,1968$.

[2] A. Farina, Antenna-based Signal Processing Techniques For Radar Systems. Artech House Boston, 1992. [Online]. Available: http: //nla.gov.au/nla.cat-vn2232434

[3] A. Farina and F. Gini, "Calculation of blanking probability for the sidelobe blanking for two interference statistical models," Signal Processing Letters, IEEE, vol. 5, no. 4, pp. 98-100, 1998.

[4] H. Finn, R. S. Johnson, and P. Z. Peebles, "Fluctuating target detection in clutter using sidelobe blanking logic," Aerospace and Electronic Systems, IEEE Transactions on, vol. AES-7, no. 1, pp. 147-159, 1971.

[5] H. L. Van Trees, Detection, Estimation, and Modulation Theory: RadarSonar Signal Processing and Gaussian Signals in Noise, 1st ed. John Wiley \& Sons, Inc., 2002, vol. 3, ch. 9, pp. 238-274.

[6] - Detection, Estimation, and Modulation Theory, 1st ed. John Wiley \& Sons, Inc., 2001, vol. 1.

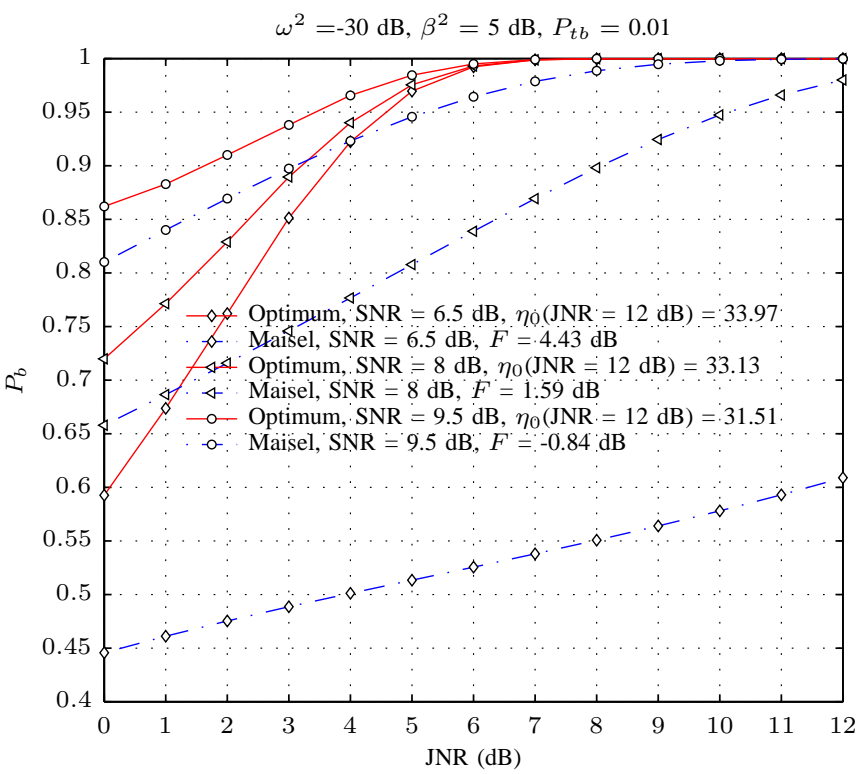

(a) $P_{t b}=0.1$

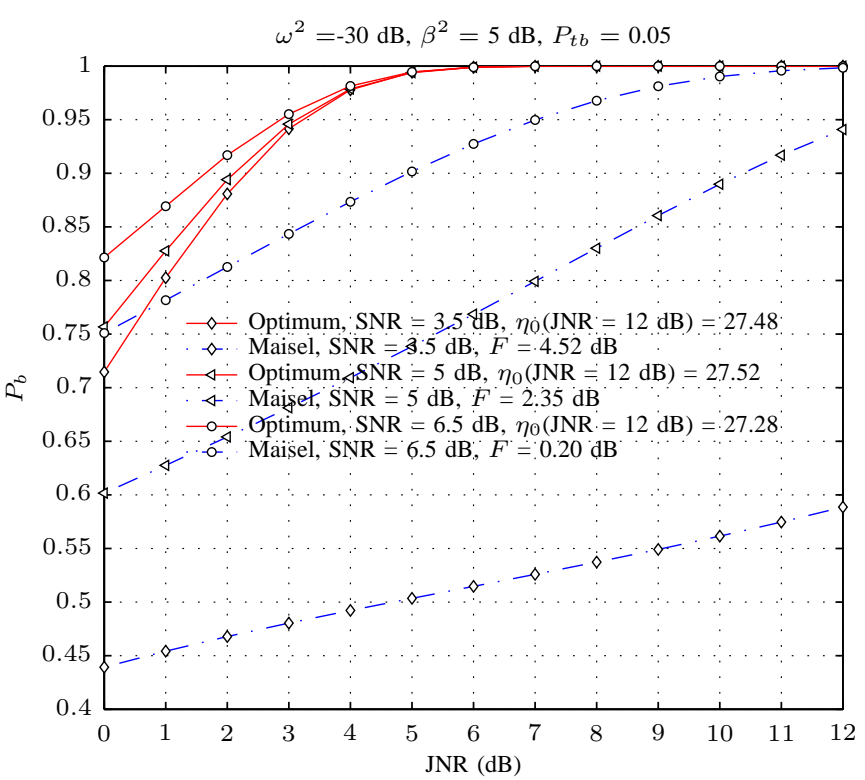

(b) $P_{t b}=0.05$

Fig. 9. Comparison of $P_{b}$ on JNR for Swerling-0 targets. Parameters: $\beta^{2}=5$ $\mathrm{dB}, \omega^{2}=-30 \mathrm{~dB}$, \# of Monte Carlo trials $=10^{6}$.

[7] K. Biyari and W. Lindsey, "Statistical distributions of Hermitian quadratic forms in complex Gaussian variables," Information Theory, IEEE Transactions on, vol. 39, no. 3, pp. 1076-1082, 1993.

[8] P. Bello and B. D. Nelin, "Predetection diversity combining with selectively fading channels," Communications Systems, IRE Transactions on, vol. 10, no. 1, pp. 32-42, 1962.

[9] U. Fernández-Plazaola, E. Martos-Naya, J. F. Paris, and J. T. Entrambasaguas, "Comments on Proakis analysis of the characteristic function of complex Gaussian quadratic forms," Computer Research Repository (CoRR), vol. abs/1212.0382, 2012.

[10] J. Proakis, "On the probability of error for multichannel reception of binary signals," Communication Technology, IEEE Transactions on, vol. 16, no. 1, pp. 68-71, 1968. 